El manejo de la información en la gestión del conocimiento gerencial universitario

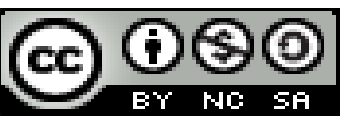

Ciencias de la educación

Artículo de investigación

\title{
El manejo de la información en la gestión del conocimiento gerencial universitario
}

\section{The management of information in the management of the university management knowledge}

A gestão da informação na gestão do conhecimento da gestão universitária

\author{
Henry F. Mendoza-Cedeño ${ }^{1}$ \\ henry.mendoza@uleam.edu.ec
}

Recibido: 10 de septiembre de 2017 * Corregido: 20 de noviembre de $2017 *$ Aceptado: 16 de diciembre de 2017

1 Magister en Contabilidad y Auditoría, Magister en Educación y Desarrollo Social, Diploma Superior en Educación Universitaria por Competencias, Ingeniero Comercial, Doctorante en la Universidad Nacional Mayor de San Marcos, Lima, Perú. 


\title{
Resumen
}

Los desafíos que se presentan en la actual Sociedad del Conocimiento es muy complejo debido a la diversidad de ideas, conceptualizaciones, estructuras mentales y estado de confort en que se encuentran las personas e instituciones, por lo que es pertinente que las Universidades asuman el reto de liderar la Gestión del Conocimiento a partir del manejo de la información. Se propone en este artículo, la evaluación del manejo de la información en la gestión del conocimiento gerencial universitario. La metodología fue de tipo La metodología fue de carácter cuantitativo con una tipología explicativa. La población estuvo compuesta por 127 gerentes y directivos universitarios de las universidades públicas de la Zona 4 del Ecuador. Entre sus resultados se pudo constatar que el estadístico $\mathrm{Fr}=14,75$ es mayor que el punto crítico $(\mathrm{X} 20,95=7,81)$, permitieron concluir que la Gestión del Conocimiento de los Directivos de la Educación Superior está siendo afectada por el Manejo de la Información, específicamente en su posición frente a los cambios estructurales, tecnológicos, sociales, culturales, ambientales y organizacionales, a pesar que en la realidad algunos de ellos no permitan enfrentar los retos.

Palabras clave: gestión del conocimiento; manejo de la información; recolección de la información; manipulación de la información; tratamiento de la información y conservación de la Información.

\begin{abstract}
The challenges arising in the current knowledge society is very complex due to the diversity of ideas, conceptualizations, mental structures and State of comfort are the people and institutions, it is relevant that the Universities take on the challenge of leading the knowledge management from the management of information. Proposed in this article, the assessment of the management of information in the management of the University management knowledge. The methodology was the methodology was quantitative with an explanatory typology. The population was composed of 127 managers and College directors of public universities of the 4 zone of Ecuador. Among its results it was found that the statistical $\mathrm{Fr}=14.75$ is greater than the critical point $(2 \mathrm{X} 0.95=7.81)$, concluded that the management of the knowledge of the directors of education higher is being affected by the handling of the information, specifically in its position on the structural, technological, social, cultural, environmental and organizational changes, though in reality some of them do not allow the challenges.
\end{abstract}


Keywords: knowledge management; information management; collection of information; manipulation of information; information processing and preservation of information.

\section{Resumo}

Os desafios apresentados na sociedade do conhecimento de hoje é muito complexa devido à diversidade de idéias, concepções, as estruturas mentais e estado de conforto que você vai encontrar indivíduos e instituições, por isso é apropriado que as universidades assumir o desafio de Liderar Gestão do Conhecimento a partir da gestão da informação. Neste artigo, propõe-se a avaliação da gestão da informação na gestão do conhecimento da gestão universitária. A metodologia foi do tipo A metodologia foi de natureza quantitativa com uma tipologia explicativa. A população era composta por 127 gerentes e diretores de universidades das universidades públicas da Zona 4 do Equador. Entre os resultados verificou-se que o Pe estatística $=14.75$ é maior do que o ponto crítico $(\mathrm{X} 20,95=7,81)$, permitiram concluir que a Gestão do Conhecimento de Administração de Ensino Superior está sendo afetado pela Gestão da Informação, especificamente em sua posição diante de mudanças estruturais, tecnológicas, sociais, culturais, ambientais e organizacionais, embora na realidade algumas delas não permitam enfrentar os desafios.

Palavras chave: gestão do conhecimento; gestão da informação; coleta de informações; manipulação de informações; processamento de informações e conservação da informação.

\section{Introducción}

Las Universidades en la actual Sociedad del Conocimiento se ven en la obligación de asumir retos, por la interacción dinámica de elementos que participan en la institución de manera organizada, utilizando los recursos y materiales, Las TIC, métodos y procesos; con la finalidad de producir, transformar y conservar conocimientos.

Siendo función de las universidades la investigación, la docencia y aprendizaje: que satisfacen los sistemas de información; los repositorios de materiales educativos, el aula virtual, las comunidades de práctica y aprendizaje. Por el cual el propósito de la Gestión del Conocimiento en la universidad será la de crear un sistema de producción, conservación, organización y circulación de conocimiento, destinado a la mejora y la efectividad de la toma de decisiones. Por tanto, surge la necesidad de valorar el Capital intelectual y de los recursos de información, para expresar con claridad su existencia, 
congregar, medir, desarrollar su uso y compartir el conocimiento, y así optimizar la calidad de los procesos que la sostienen.

En un mundo de cambios frecuentes en que se desenvuelve las sociedades producto de la globalización, la era del conocimiento y de la información se hace imperante que las empresas tanto públicas como privadas busquen su desarrollo integral y competitivo, a través de optimizar sus recursos y aplicar estrategias que cedan al logro de objetivos empresariales y personales.

El incremento económico descansa cada día en la calificación de las personas, descrito en habilidades para concebir innovaciones tecnológicas, incorporar en redes dinámicas, que beneficia a quienes cuentan con dichas capacidades. Frente a la exigencia de una mano de obra capaz de adquirir nuevas calificaciones y adecuarse a los vertiginosos cambios tecnológicos durante su vida laboral, por lo que los regímenes de educación y formación deben ajustarse a estos nuevos requerimientos, en este sentido el presente artículo evalúa el manejo de la información en la gestión del conocimiento gerencial universitario.

\section{Desarrollo}

Una de las formas a considerar para indagar es estar conscientes de los conocimientos que nos interesa saber. Se habla que vivimos en la era del conocimiento, donde la búsqueda se hace complejo en un mar de datos, por lo se necesitan estrategias de indagación para conocer sobre un asunto de interés. El conocimiento de acuerdo a (Steinberg, 1996) citado por Estela (2013), es saber mucho más que conocer. Es un conocimiento que no se olvida, que no reside sencillamente en la superficie externa de la memoria sino en los pliegues más profundos del verdadero ser. Lo que se sabe se convierte en parte de uno mismo. Y así como uno mismo siempre «es», aunque no sea igual de niño que de adulto, la Sabiduría obliga a ese avance en madurez que corre con el Tiempo, si bien un tiempo que se hace eterno cuando se refiere al propio crecimiento.

\section{La Gestión del Conocimiento}

Los revolucionarios reconocen que la competitividad ya no es cuestión de diferenciarse en productos o servicios, sino de competir con un concepto diferente del negocio (William Gates (CEO Microsoft). Desde las perspectivas de las Organizaciones, el conocimiento está definido como la información que tiene valor para ella, es decir, aquella información que permite originar acciones asociadas a buscar 
como satisfacer las demandas del mercado y apoyar las nuevas oportunidades por medio de la explotación de las competencias centrales de la Organización. Es decir, la identificación de la información que la organización requiere para satisfacer sus objetivos.

La Gestión del Conocimiento, según Darío (2004), se define como el conjunto de procesos, estructuras organizativas, aplicaciones y tecnologías, a través de las cuales una empresa recoge, ordena, analiza, comparte y difunde, de manera consciente, su conocimiento entre el mayor número de empleados para aprovecharlo en beneficio de su organización

\section{Manejo de la Información}

Información arma muy poderosa, que, si cae en manos de personas incorrectas, puede tener graves consecuencias el propietario de la información. Razón de suma importancia dar el sumo cuidado a toda clase de información de las personas, pues lo que puede parecer insignificante para algunos, para otros puede servir para realizar alguna avería. Asimismo, es de recomendación realizar una clasificación de la información para tomar medidas de seguridad según sea necesario. Se debe seleccionar correctamente la información crítica, y darle la seguridad adecuada, manejar el riesgo y evitar fugas de información. Proteger la información delicada a través del respaldo en un servidor externo o un medio de almacenamiento externo. Realizar los respaldos de información en por lo menos 2 medios diferentes. Así mismo evitar eliminar la información útil hasta cerciorarse que se encuentra debidamente protegida. Además, evitar dejar los documentos importantes en lugares visibles o de fácil acceso para otras personas.

\section{Características del Manejo de la Información}

Es un proceso dinámico: porque está en continuo movimiento, inevitable: porque se requiere para la transmisión de significados, irreversible: porque una vez realizada, no puede regresar, borrarse o ignorarse. Bidireccional: porque existe una respuesta en ambas direcciones. Verbal y no verbal: porque implica la utilización de ambos lenguajes. Con esta información podemos entender cómo es que hoy en día internet se volvió en un medio de comunicación tan importante ya que permite comunicarnos permanentemente y en forma instantánea. (Javkin, 2007)

Los enlaces online y la introducción de la tecnología muy útil para la sociedad, siempre que se utilice de manera correcta, conociendo elegir qué información debe compartir y cual no, que datos mostrar y 
cuales ocultar, y esencialmente saber mantener la privacidad, la intimidad, resguardando la seguridad, y la ética de los usuarios y consumidores de la información online. En el manejo de la información, plantea Odrizola. (2000), se caracterizan los siguientes elementos; la recolección, manipulación, tratamiento y conservación de la información.

Durante el manejo de la información, se pueden utilizar herramientas informáticas a través de la computadora como: procesador de texto, la base de datos, graficadores, correo electrónico, hojas de cálculo, buscadores, programas de diseño, presentadores, etc., Asimismo un conocimiento de la computadora y de sus elementos, objetos que manejan y operaciones básicas. La Internet conecta a todo el mundo, las extranet conectan a las empresas entre sí y las Intranets conectan a los particulares con las empresas. Los nuevos competidores dentro del Internet podrán llegar de cualquier parte a robarnos los clientes. En las grandes corporaciones, aparecen medidas universales y abiertas para intercambiar información, a través de las intranets provoca equipos de trabajo cruzado y acelera el ocaso de estructuras escalonadas de sus sistemas de información preferencial. (IMIPE, s/f).

La manipulación de la información, ha de ser manejada de forma adecuada, por otro lado, hay que recalcar que el uso de los documentos sin precaución, puede ocasionar graves deterioros físicos al material y a la información que contienen por lo que se debe embalar y tener una protección según las instrucciones.

Durante el tratamiento y conservación de la información, la documentación al estar expuestos a factores y mecanismos de variación sufre cambios en su composición física, que afectan su función y ponen en peligro la información. Por lo que es necesario el cuidado preventivo y adecuado de los documentos en el archivo. Serán los funcionarios a cargo de la organización, sistematización y facilitación de archivos, quienes tienen la responsabilidad de garantizar la perdurabilidad del acervo documental. Son las medidas preventivas de conservar y preservar los archivos en relación con el medio ambiente, las condiciones de almacenamiento adecuado, uso y manipulación.

\section{Metodología.}

La metodología fue de carácter Cuantitativo con una tipología explicativa. La población estuvo compuesta por el total de 30 Autoridades y Miembros del equipo directivo que participan en el proceso de acreditación de las universidades públicas de la Zona 4 de Ecuador. Además, se complementó con 
los funcionarios especialistas en materia de gestión del conocimiento que alcanzaron a 125 especialistas de entre los docentes de: las Universidades y la Escuela Superior en estudio. Por lo tanto, la población alcanzó a 155 profesionales de los cuales posterior al cálculo de la muestra quedo constituido por 127 del total. El instrumento utilizado fue tipo cuestionario y la técnica la encuesta. La estadística utilizada fue la descriptiva y la Prueba Friedman (Fr) expuesta en Wakerly (2008).

\section{Resultados.}

Se presentan en tablas con sus respectivos gráficos el análisis del manejo de la información en la gestión del conocimiento del gerente universitario obtenida a través del instrumento aplicado a gerentes universitarios de la Zona 4 del Ecuador.

Se presenta en la tabla y grafico 1, posterior al análisis de los datos que evaluaron la recolección en el manejo de la información durante la gestión del conocimiento, que el 54\% del total de los directivos encuestados coincidieron en opinar que concuerdan fuertemente en afirmar que durante la gestión del conocimiento se recolecta información como apoyo por parte de los directivos de la educación superior. Seguido del $45 \%$ que consideraron la alternativa concuerdan, cabe mencionar que solo el $1 \%$ del total de los directivos, opino estar en discrepancia fuertemente. Se destaca el hecho, en la cual no se evaluaron repuestas para las alternativas no opina y discrepa.

Tabla 1. Recoleccion de la información

\begin{tabular}{|l|c|c|}
\hline \multicolumn{1}{|c|}{ Respuesta } & Frecuencia & Porcentaje \\
\hline Concuerda Fuertemente (CF) & 69 & $54 \%$ \\
\hline Concuerda (C) & 57 & $45 \%$ \\
\hline No opina (No) & 0 & $0 \%$ \\
\hline Discrepa (D) & 0 & $0 \%$ \\
\hline Discrepa Fuertemente (DF) & 127 & $1 \%$ \\
\hline Total & $\mathbf{1 2 7}$ & $\mathbf{1 0 0 \%}$ \\
\hline
\end{tabular}

Fuente: elaboración propia. 


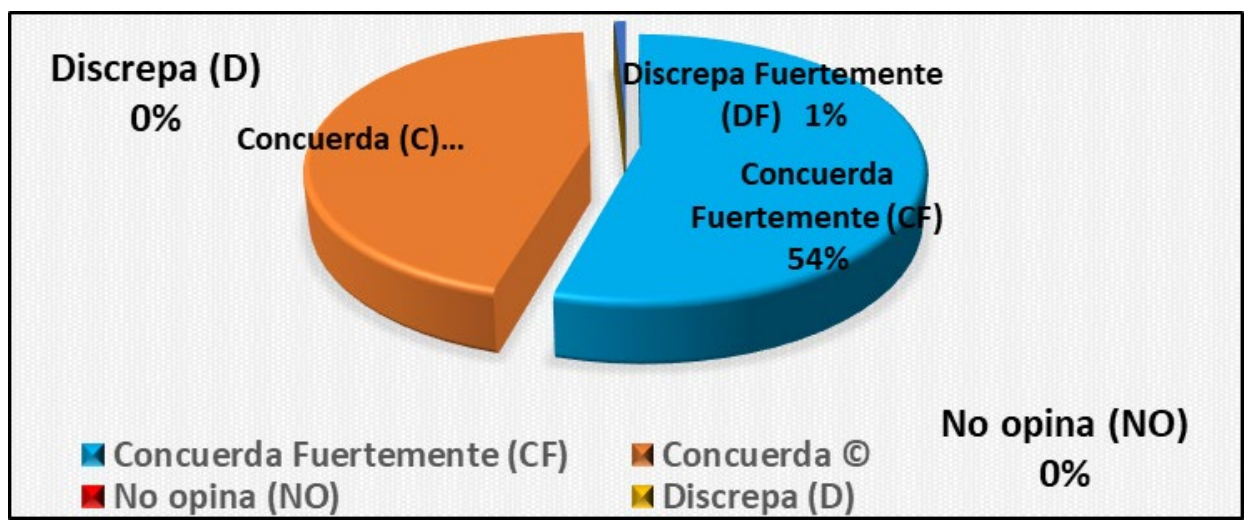

Grafico 1. Recoleccion de la informacion

Se presenta en la tabla y grafico 2, posterior al análisis de los datos que midieron la manipulación en el manejo de la información durante la gestión del conocimiento, que el 51\% del total de los directivos encuestados coincidieron en opinar que concuerdan que durante la gestión del conocimiento se manipula la información como apoyo por parte de los directivos de la educación superior. Seguido del $47 \%$ que consideraron la alternativa concuerdan fuertemente, cabe mencionar que solo el $2 \%$ del total de los directivos, opino estar en discrepancia fuertemente. Se destaca el hecho, en la cual no se evaluaron repuestas para las alternativas no opina y discrepa.

Tabla 2. Manipulación de la información.

\begin{tabular}{|l|c|c|}
\hline \multicolumn{1}{|c|}{ Respuesta } & Frecuencia & Porcentaje \\
\hline Concuerda Fuertemente (CF) & 60 & $47 \%$ \\
\hline Concuerda (C) & 65 & $51 \%$ \\
\hline No opina (No) & 0 & $0 \%$ \\
\hline Discrepa (D) & 0 & $0 \%$ \\
\hline Discrepa Fuertemente (DF) & 2 & $2 \%$ \\
\hline Total & $\mathbf{1 2 7}$ & $\mathbf{1 0 0 \%}$ \\
\hline
\end{tabular}

Fuente: elaboración propia. 
Grafico 2. Recoleccion de la informacion.

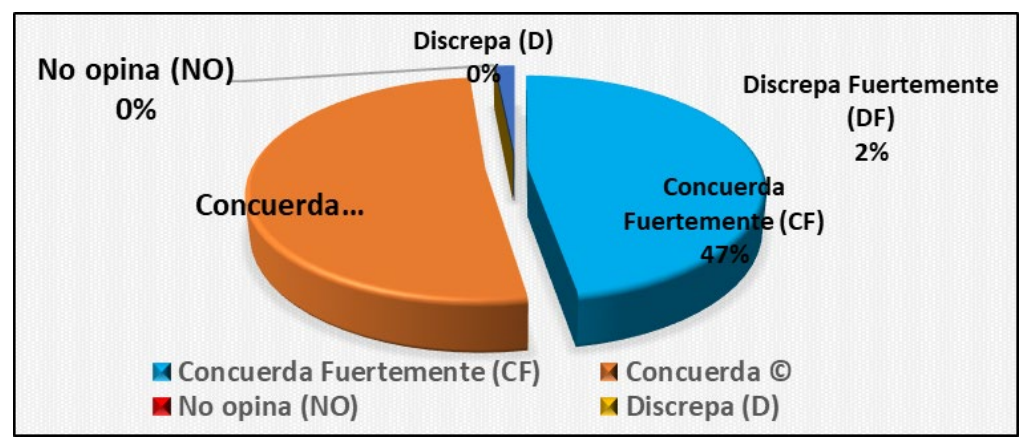

Se presenta en la tabla y grafico 2, posterior al análisis de los datos que midieron la manipulación en el manejo de la información durante la gestión del conocimiento, que el 51\% del total de los directivos encuestados coincidieron en opinar que concuerdan que durante la gestión del conocimiento se manipula la información como apoyo por parte de los directivos de la educación superior. Seguido del 47\% que consideraron la alternativa concuerdan fuertemente, cabe mencionar que solo el 2\% del total de los directivos, opino estar en discrepancia fuertemente. Se destaca el hecho, en la cual no se evaluaron repuestas para las alternativas no opina y discrepa.

Tabla 3. Tratamiento de la información

\begin{tabular}{|l|c|c|}
\hline \multicolumn{1}{|c|}{ Respuesta } & Frecuencia & Porcentaje \\
\hline Concuerda Fuertemente (CF) & 67 & $53 \%$ \\
\hline Concuerda (C) & 59 & $46 \%$ \\
\hline No opina (No) & 0 & $0 \%$ \\
\hline Discrepa (D) & 0 & $0 \%$ \\
\hline Discrepa Fuertemente (DF) & 1 & $1 \%$ \\
\hline Total & $\mathbf{1 2 7}$ & $\mathbf{1 0 0 \%}$ \\
\hline
\end{tabular}

Fuente: elaboración propia. 
El manejo de la información en la gestión del conocimiento gerencial universitario

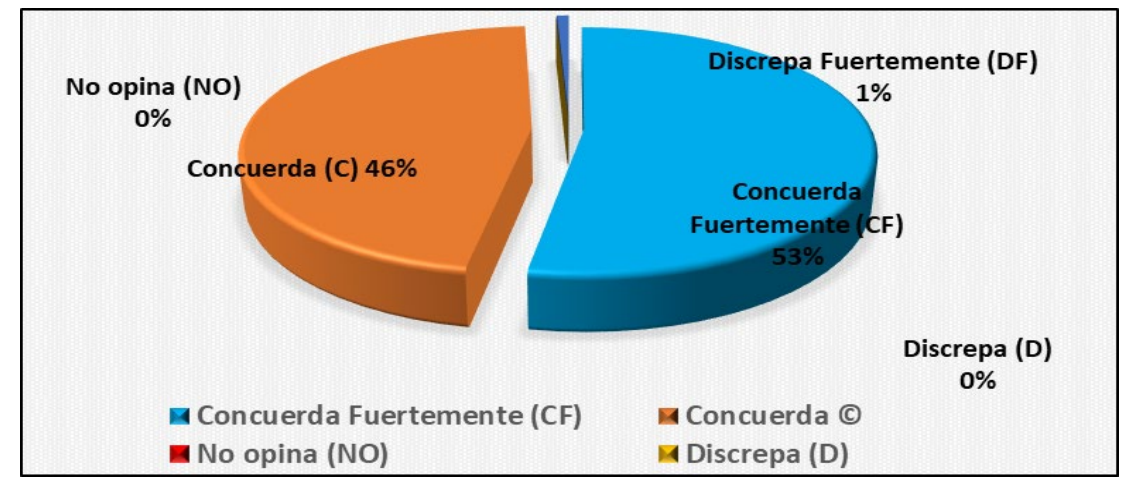

Grafico 3. Tratamiento de la información

Se presenta en la tabla y grafico 4, posterior al análisis de los datos que evaluaron la conservación de la información en la gestión del conocimiento, que el 53\% del total de los directivos encuestados coincidieron en opinar que concuerdan fuertemente que durante la gestión del conocimiento se conserva información como apoyo por parte de los directivos de la educación superior. Seguido del $42 \%$ que consideraron la alternativa concuerdan. Cabe destacar que una gran minoría representada por; el $4 \%$ de los directivos que no están de acuerdo, el 2\% discrepan y el $2 \%$ discrepan fuertemente. Asimismo, el 1\% de los directivos está indeciso.

Tabla 4. Conservación de la información

\begin{tabular}{|l|c|c|}
\hline \multicolumn{1}{|c|}{ Respuesta } & Frecuencia & Porcentaje \\
\hline Concuerda Fuertemente (CF) & 67 & $53 \%$ \\
\hline Concuerda (C) & 53 & $42 \%$ \\
\hline No opina (No) & 1 & $1 \%$ \\
\hline Discrepa (D) & 3 & $2 \%$ \\
\hline Discrepa Fuertemente (DF) & 3 & $2 \%$ \\
\hline Total & $\mathbf{1 2 7}$ & $\mathbf{1 0 0 \%}$ \\
\hline
\end{tabular}

Fuente: elaboración propia. 


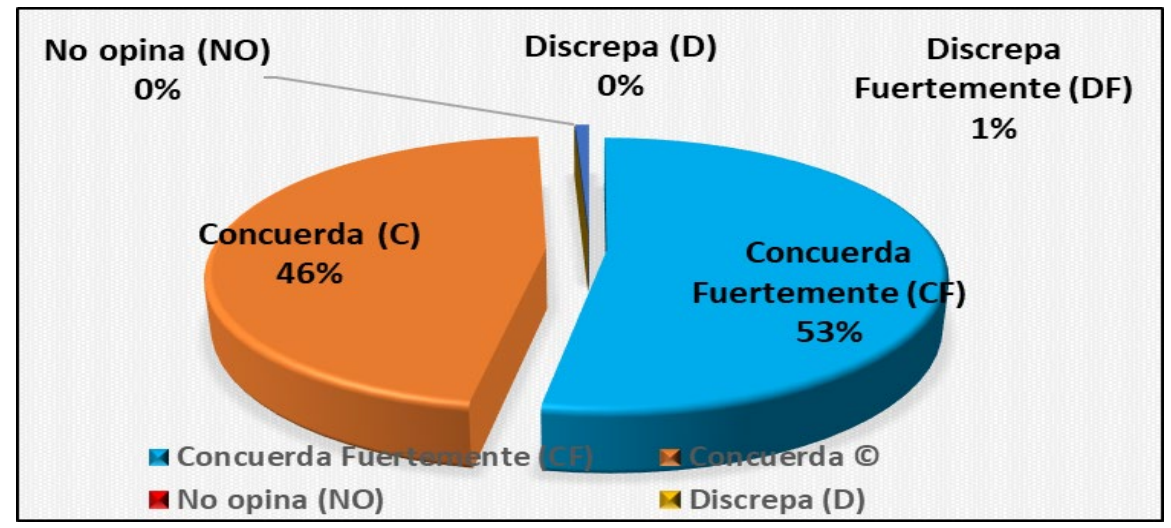

Grafico 4. Conservación de la información

En los resultados del análisis de la prueba de Hipótesis de Friedman (Fr), en la que se constató que el estadístico $\mathrm{Fr}=14,75$ es mayor que el punto crítico $(\mathrm{X} 20,95=7,81)$, es decir que la gestión del conocimiento está siendo afectado por el manejo de la información por parte de los Directivos de la Educación Superior de las Universidades Públicas de la Zona 4 del Ecuador. 


\section{Prueba Friedman (Fr).}

$$
\mathbf{F r}=\frac{12}{k n(n+1)}\left[\sum R^{2}\right]-3 k(n+1)
$$

Para: $\alpha=0,05 ; \mathrm{Gl}=\mathrm{k}-1=4-1=3 \quad \mathrm{X}_{0,95}^{2}=7,81$

\begin{tabular}{|c|c|c|c|c|c|c|c|c|c|}
\hline \multicolumn{2}{|c|}{ Recolección } & \multicolumn{2}{|c|}{ Manipulación } & \multicolumn{2}{|c|}{ Tratamiento } & \multicolumn{2}{|c|}{ Conservación } & \multicolumn{2}{|c|}{$\begin{array}{l}\text { Sumas del } \\
\text { orden }\end{array}$} \\
\hline Resp. & Drden & Resp. & rden & Resp. & Resp. & Orden & Resp. & Suma & Cuadrado \\
\hline 69 & 5 & 60 & 5 & 67 & 5 & 67 & 5 & 20,0 & 400,00 \\
\hline 57 & 4 & 65 & 4 & 59 & 4 & 53 & 4 & 16,0 & 256,00 \\
\hline 0 & 1,5 & 0 & 1,5 & 0 & 1,5 & 1 & 1 & 5,5 & 30,25 \\
\hline 0 & 1,5 & 0 & 1,5 & 0 & 1,5 & 3 & 2,5 & 7,0 & 49,00 \\
\hline 1 & 3 & 2 & 3 & 1 & 3 & 3 & 2,5 & 11,5 & 132,25 \\
\hline & & & & & & & & $\Sigma$ & 867,50 \\
\hline
\end{tabular}

$$
\begin{gathered}
\mathbf{F r}=\frac{12}{4(5)(5+1)}[867,50]-3(4)(5+1) \\
\mathbf{F r}=(12 / 120) 867,50-72=14,75
\end{gathered}
$$

\section{Conclusiones}

Se constató que la Gestión del Conocimiento de los Directivos de la Educación Superior está siendo afectada por el Manejo de la Información, específicamente con los relacionados a los cambios estructurales, tecnológicos, sociales, culturales, ambientales y organizacionales, a pesar que en la realidad alguna de ellos no permitan enfrentar los retos.

El mayor porcentaje refirió que concuerdan fuertemente, en que la recolección de la información durante su manejo sirve de apoyo durante la gestión del conocimiento, entre los Directivos de la Educación Superior investigados. 
Concuerdan en opinión de los Directivos de la Educación Superior investigados que la manipulación de la información durante su manejo sirve de apoyo durante la gestión del conocimiento.

Concuerdan fuertemente en opinión de los Directivos de la Educación Superior investigados que que durante la gestión del conocimiento se trata la información como apoyo en la gestión del conocimiento.

Los datos que evaluaron la conservación de la información en la gestión del conocimiento, constato entre los directivos encuestados mayor coincidencia de opiniones en la alternativa concuerdan fuertemente que durante la gestión del conocimiento se conserva información como apoyo por parte de los investigados.

\section{Referencias Bibliográficas}

Dario, P. M. (2004). Los modernos alquimistas. Epistemologia corporativa y gestion del conocimiento. Medellín: Fondo Editorial Universidad EAFIT.

Estela, A. E. (2013). Lineamientos prácticos para investigar. Lima: ESTELA Hnos. S.R.L.

Gates. (1999). Los negocios en la eraa digital.

IMIPE. (s/f). Manual de preservación conservación y tratamiento de los documentos del archivo.

$\begin{array}{llll}\text { Retrieved febrero } & 28, & \text { from }\end{array}$ http://www.transparenciamorelos.mx/sites/default/files/Manual\%20de\%20Conservaci\%C3\%B3n\%2 0y\%20Tratamiento\%20del\%20archivo\%20de\%20concentraci\%C3\%B3n_0.pdf:

http://www.transparenciamorelos.mx/sites/default/files/Manual\%20de\%20Conservaci\%C3\%B3n\%2 0y\%20Tratamiento\%20del\%20archivo\%20de\%20concentraci\%C3\%B3n_0.pdf

Javkin, A. (2007, noviembre 7). Blog de Ciencia, Tecnología y Sociedad. Una herramienta para pasar "del trabajo solitario al solidario". Retrieved marzo 5, 2017, from http://cuandotodossaben.blogspot.pe/2008/11/manejo-de-la-informacin.html:

http://cuandotodossaben.blogspot.pe/2008/11/manejo-de-la-informacin.html

Odrizola. (2000). Variables de decisión y factores clve en el roceso información y conocimiento.

Wakerly, D. D. (2008). Estadística Matemática con Aplicaciones. México: Latinoamericana S. A. 